INFORMATION

\title{
COLLOQUE « CONDORCET »
}

Savant, philosophe et homme politique de la Révolution, l'encyclopédiste Condorcet est une figure à la fois exemplaire et originale. Cependant, des aspects importants de son œuvre sont encore mal connus ou mal interprétés. Ce colloque, moment essentiel d'un travail pluridisciplinaire systématique, devrait permettre de réévaluer le rôle historique de Condorcet et, à travers lui, de reprendre la question des rapports entre les philosophes, les savants et la Révolution.

\section{LES THËMES}

(Il s'agit d'une liste, non exhaustive, de sujets qui seront abordés - ou qu'il serait souhaitable d'aborder - lors du colloque, toute autre proposition étant bienvenue.)

1. Mathématiques pures - Mécanique céleste :

- Réévaluation des travaux de Condorcet en mathématiques pures : calcul intégral (création de la théorie de l'intégration en termes finis), équations algébriques ; leur originalité et leur importance pour la compréhension de sa pensée.

- Système du monde, problème des trois corps et théorie des comètes.

2. Probabilités - Statistiques - Mathématique sociale :

- Inventaire précis de l'apport de Condorcet à la théorie mathématique des probabilités.

- Le rôle du théorème de Bayes dans sa conception des probabilités.

- De l'arithmétique politique à la mathématique sociale.

- Statistiques, tableaux et classifications.

- Choix social et problème de l'agrégation.

- Théorie des élections.

- Probabilités et calculs financiers : assurances, rentes viagères, loteries, droits féodaux.

- Le problème de la protection sociale.

Revue de synthèse : IVe S. N 1 , janv.-mars 1987. 
3. Science, Savants et Société :

- Science et politique au moment du ministère Turgot.

- Nouveau rôle de la science et des savants dans la société.

- Controverses et conflits à l'Académie.

- La question des poids et mesures ; celle des monnaies.

- Condorcet historien des sciences à travers ses Éloges académiques.

- Les contributions de Condorcet à l'œuvre encyclopédique.

- Le contenu scientifique de l'enseignement.

- Politique de la science et science de la politique.

4. Économie :

- Influence de Turgot sur Condorcet.

- Condorcet et Adam Smith.

- De l'économie politique à la politique économique.

- Condorcet libéral ?

- Condorcet et le thème de la croissance.

5. Politique :

- Condorcet et la révolution d'Amérique.

- Ses écrits sur les États généraux et les Assemblées provinciales.

- Condorcet face aux Déclarations des droits de l'Homme.

- Sa notion de souveraineté : élitisme scientifique et exigence démocratique, évolution de ce conflit de 1786 à 1793.

- Condorcet à la Législative : son projet d'Instruction publique ; Condorcet à la première Commission des douze (juin/septembre 1792).

- Condorcet à la Convention : le problème de la Constitution ; Condorcet girondin ou inclassable?

- Condorcet journaliste.

- Condorcet et les droits des femmes.

- Condorcet contre l'esclavage des Noirs.

6. Philosophie :

- Sa théorie de la connaissance.

- Représentation tabulaire et statut de l'histoire et du progrès chez Condorcet : l'Esquisse et les matériaux du Tableau historique.

- Condorcet et le problème de la langue.

- Sa pensée juridique.

- Fondements et conséquences philosophiques de son projet d'Instruction publique.

- Condorcet et les philosophes.

- Condorcet et la question religieuse.

Ce découpage disciplinaire (correspondant à six demi-journées du colloque) devrait permettre d'aborder le maximum d'aspects de la pensée et de l'action de Condorcet. Mais il a, pour l'encyclopédiste, un caractère en partie arbitraire qu'il s'agira de dépasser en abordant (notamment lors d'une quatrième journée, de synthèse) quelques questions globales, telles que : 
- Y a-t-il ou non unité de la pensée de Condorcet et si oui, comment la caractériser ?

- Comment articuler les divers aspects de son œuvre dans une biographie ? (évolution de sa pensée et problème de périodisation).

- Quelle a été la réception de l'œuvre de Condorcet auX $\mathrm{XIX}^{\mathbf{e}}$ et $\mathrm{XX}^{\mathrm{e}}$ siècles ? Comment expliquer l'alternance de l'influence (idéologues, positivistes, républicains de la III' République, etc.) et de l'oubli, des jugements positifs et négatifs, jusqu'à nos jours ?

- En quoi le cas de Condorcet permet-il de mieux comprendre les rapports complexes entre le mouvement des Lumières et la Révolution ?

\section{ORGANISATION DU COLLOQUE}

Ce colloque international se tiendra à Paris en juin 1988. Sa durée sera de quatre jours. Il est prévu une publication des Actes du colloque.

\section{- Comité d'organisation du colloque :}

E. Brian, B. Bru, M. Chapront, A.-M. Chouillet, M. Crampe-Casnabet, P. Crépel, G. Faccarello, S. Farandjis, C. Gilain, F. Hincker, C. Houzel, C. Kintzler, P. Michaud, B. Monjardet, M. Morange, M. Paty, J.-C. Perrot, R. Rashed.

\section{- Patronage scientifique du colloque :}

Équipe REHSEIS (Recherches épistémologiques et historiques sur les sciences exactes et les institutions scientifiques) du C.N.R.S.,

Centre international de synthèse,

Institut d'histoire de la Révolution française,

Société française d'étude du XVIII' siècle (Premières institutions).

\section{- Préinscription :}

Pour une bonne organisation du colloque, nous demandons à tous les collègues intéressés de nous transmettre dès maintenant leurs nom, prénom, adresse et le sujet sur lequel ils pourraient intervenir. Nous leur ferons parvenir alors des renseignements supplémentaires.

Le courrier est à adresser à :

Pierre Crépel et Christian Gilain

"Colloque Condorcet "

Équipe REHSEIS

Université Paris VII

2, place Jussieu

75251 Paris Cedex 05. 